\title{
Gestión del suministro para disponibilidad de medicamentos e insumos en micro redes de salud 2020
}

Támara Cruz Peña Piñan

tamaracpp@ @otmail.com

Escuela de posgrado

Universidad César Vallejo

ORCID: 0000-0002-5897-2377

José Manuel Delgado Bardales

jmdelgadob@ucvvirtual.edu.pe

Escuela de posgrado

Universidad César Vallejo

ORCID:0000-0001-6574-2759

Scopus autor ID: 24070333700

Código Renacyt: P0050554

\section{RESUMEN}

La presente investigación tuvo como objetivo, Gestión del suministro para disponibilidad de medicamentos e insumos en microredes de salud 2020, la investigación fue cualitativa de diseño revisión sistemática, se realizó el análisis de 10 artículos científicos relacionados a la variable de estudio. Se encontró que los artículos científicos, enfocados a la gestión del suministro para disponibilidad de medicamentos e insumos en microredes de salud pertenecen al tipo y diseño de estudios descriptivos. Los artículos científicos, gestión de suministros, corresponde a diferentes bases de datos, todos ellos corresponden al tipo y diseño de estudios descriptivos. Posterior a la revisión sistémica, se concluye que el $60 \%$ de los estudios relacionados que los estilos de consumo influyen en la vida sostenible en el contexto de la crisis económica, debido a que los estilos de consumo demandan políticas, condiciones y consecuencias de contaminación básicamente; y se puede establecer que el consumo tiene un impacto social lógicamente distinto en los diferentes países del mundo. Por otro lado, el 50\% concluye que los estilos de vida son importantes porque el comportamiento de las personas depende de ello que establezca un adecuado consumo. Así mismo las consecuencias del consumo no disminuyó muy por lo contrario sino no tomamos atención va en incremento. Las personas para que el consumo tome un camino diferente a favor de la vida sostenible a pesar de la crisis económica de los países, porque hay acciones que no depende de dinero sino del comportamiento social de las personas.

Palabras clave: Gestión del suministro, disponibilidad de medicamentos, insumos. 


\title{
Supply management for the availability of medicines and supplies in health micro-networks 2020
}

\begin{abstract}
The objective of this research was, Supply management for the availability of medicines and supplies in health micro-networks 2020, the research was qualitative with a systematic review design, the analysis of 10 scientific articles related to the study variable was carried out. It was found that the scientific articles, focused on the management of the supply for the availability of medicines and supplies in health micro-networks belong to the type and design of descriptive studies. The scientific articles, supply management, correspond to different databases, all of them correspond to the type and design of descriptive studies. After the systemic review, it is concluded that $60 \%$ of the studies related that consumption styles influence sustainable life in the context of the economic crisis, due to the fact that consumption styles demand policies, conditions and consequences of pollution basically; and it can be established that consumption has a logically different social impact in different countries of the world. On the other hand, $50 \%$ conclude that lifestyles are important because people's behavior depends on it to establish adequate consumption. Likewise, the consequences of consumption did not decrease, on the contrary, if we did not pay attention, it is increasing. People for consumption to take a different path in favor of sustainable life despite the economic crisis in the countries, because there are actions that do not depend on money but on the social behavior of people.
\end{abstract}

Keywords: Supply management, availability of drugs, supplies

Artículo recibido: 03 nov. 2020 Aceptado para publicación: 07 dic. 2020 Correspondencia tamaracpp@ hotmail.com Conflictos de Interés: Ninguna que declarar 


\section{INTRODUCCIÓN}

La Organización Mundial de la Salud -OMS (2011) indica que los gobiernos nacionales deben asegurar que los pacientes reciban los medicamentos de acuerdo con las necesidades clínicas, en las dosis y tiempo adecuados, y al menor costo posible. Los medicamentos e insumos son considerados bienes sociales que intervienen en la recuperación de la salud y la prevención de enfermedades, donde todas las personas tienen acceso como cumplimiento de su pleno derecho; de acuerdo con el Consejo de Derechos de las Naciones Unidas (2013), la Organización Internacional de Salud Management Sciences for Health (2002) y el Congreso de la República del Perú (1993); donde es necesario el abastecimiento y disponibilidad constante de los medicamentos, por su vital importancia en la actividad sanitaria y con ello salvaguardar el adecuado funcionamiento de los servicios de salud públicos, e incluso los privados.

La mayor parte de las principales causas de muerte y discapacidad en los países en desarrollo pueden evitarse, tratarse o por lo menos aliviarse con medicamentos esenciales eficaces en relación con el costo. A pesar de ello, centenares de millones de personas no tienen acceso a los medicamentos esenciales. El suministro de medicamentos esenciales según Salazar (2014) menciona que el sistema de abastecimiento público de medicamentos en el Perú juega un rol importante en la provisión de servicios de salud de calidad, por ello requiere que su gestión sea eficaz y eficiente, además dice que debe estar en concordancia con las políticas de modernización de la gestión pública y la reforma del sector salud. A nivel internacional, en El Salvador se reporta que la gestión integral de suministro de medicamentos e insumos de salud sigue los procesos de Selección, Estimación, adquisición conjunta, Almacenamiento, Disposición final, Distribución, Promoción del uso racional y Farmacovigilancia; fundamentados en los tres pilares de la política farmacéutica nacional: acceso, uso y calidad (Ministerio de Salud, 2012, p. 19).

Según Acuña (2014) en la encuesta de hogares de los países Guatemala, Honduras y Nicaragua se evidencia que los fenómenos de la falta de acceso a los servicios de salud y a medicamentos no son independientes, así se encontró que respecto a la falta de acceso a medicamentos afectó a cerca de $21 \%$ de los hogares. Asimismo, respecto a la disponibilidad de los medicamentos esenciales en los establecimientos de salud de 
atención primaria el Ministerio de Salud del Perú (2018) en sus indicadores de disponibilidad señala que constituye un elemento fundamental para garantizar el acceso de la población a los mismos, además nos dice que, la medición de ellos, permite conocer la eficiencia de la gestión de los sistemas de suministro y un indicador de igual o mayor a $90 \%$ se considera disponibilidad óptima.

Espinoza (2017) señala que los centros y puestos de salud del primer nivel de atención pública de la región Junín presentan una ineficiente gestión en el abastecimiento y control del stock de medicamentos que ocasiona un sobre costo en el almacenaje de los mismos. El 32\% de los establecimientos carecen de medicamentos básicos para la atención en obstetricia y neonatal primaria. El $71 \%$ presenta un sub stock en medicamentos primordiales ( $p$ 207). Es común escuchar de los problemas de abastecimiento y uso de medicamentos en los establecimientos de salud públicos del Perú. Sin embargo, ¿sabemos por qué ocurre esta situación? La respuesta más sencilla y en la que solemos caer la mayoría de personas es atribuir la causa a mala gestión. Sin embargo, esta es una simplificación que no refleja realmente la complejidad y los problemas del sistema de compra, almacenamiento y distribución de medicamentos en nuestro país (Salomón, 2017).

En tal sentido, en el Centro de Salud Morales que actúa como cabecera de la microred urbana de San Martín, por los problemas identificados a través de la presente investigación busca demostrar que, con una gestión eficiente del suministro de medicamentos e insumos, con el compromiso social, se logre conseguir su disponibilidad en los establecimientos de salud de la micro red Morales. Por lo mencionado se formuló como problema: ¿Cómo debe ser la gestión del suministro para la disponibilidad de medicamentos e insumos en micro redes de salud 2020? Y como Objetivo: Identificar las características de la gestión de suministro para la disponibilidad de medicamentos e insumos en micro redes de salud 2020.

\section{ESTRATEGIAS METODOLÓGICAS O MATERIALES Y MÉTODOS}

El tipo de investigación es básica de diseño descriptivo con revisión sistemática, las revisiones sistemáticas son investigaciones científicas en las cuales la unidad de análisis son los estudios originales primarios, constituyen una herramienta esencial para sintetizar la información científica disponible, incrementar la validez de las conclusiones de estudios individuales e identificar áreas de incertidumbre donde sea necesario realizar 
investigación (Hernández, 2016, p.217). Es descriptiva, porque se recogió información relacionada a la gestión de suministro para la disponibilidad de medicamentos e insumos en microredes de salud (Tamayo y Tamayo, 2009, p.54). Por lo mencionado, se revisó información científica y se visualizó como solucionar una necesidad en la organización de la entidad pública, para mejorar la gestión de suministro para la disponibilidad de medicamentos e insumos en micro redes de salud y otras deficiencias existentes.

La recolección de datos se realizó a través de una tabla de registro de datos y la fuente fue revisión bibliográfica de artículos de investigaciones tanto nacionales como internacionales que tuvieron como tema principal la gestión de suministro para la disponibilidad de medicamentos e insumos en microredes de salud; de todos los artículos que se encontraron, se incluyeron los estudios (10) más importantes según nivel de evidencia y se excluyeron los menos relevantes. Se estableció la búsqueda siempre y cuando se tuvo acceso al texto completo del artículo científico.

La técnica de análisis se desarrolló a través del análisis de la revisión sistemática evaluando cada uno de los artículos para una comparación de los puntos o características en las cuales concuerda y los puntos en los que existe discrepancia entre artículos nacionales e internacionales. Además, de acuerdo a criterios técnicos pre establecidos, se realizó una evaluación crítica e intensiva de cada artículo, a partir de ello, se determinó la calidad de la evidencia y la fuerza de recomendación para cada artículo 


\section{RESULTADOS Y DISCUSIÓN}

\section{Resultados}

Tabla 1: Gestión del suministro para disponibilidad de medicamentos e insumos en microredes de salud 2020

\section{DATOS DE LA PUBLICACIÓN}

\section{Autor Año Nombre de la Investigación}

Aguas, J., Jaimes, W.,

\& Escamilla, E.
Coordinación en redes de suministro de medicamentos.

caso aplicado al sector salud colombiano.
Revista donde se ubica la Publicación

Revista Colombia.

EIA, doi:http://dx.doi.org/10.14 Vol. 13(25), 171-183. 508/reia.2016.13.25.171-183

\section{CONTENIDO DE LA PUBLICACIÓN}

\begin{tabular}{|c|c|c|c|c|c|}
\hline $\begin{array}{c}\text { Tipo y Diseño } \\
\text { de } \\
\text { Investigación }\end{array}$ & $\begin{array}{l}\text { Población } \\
\text { y Muestra }\end{array}$ & Instrumentos & Aspectos étic & Resultados & Conclusión \\
\hline $\begin{array}{l}\text { Descriptiva } \\
\text { estudio de caso }\end{array}$ & $\begin{array}{l}\text { Centros } \\
\text { hospitalario } \\
\mathrm{s} \text { y } \\
\text { Documento } \\
\mathrm{s}\end{array}$ & $\begin{array}{l}\text { Ficha de } \\
\text { recolección } \\
\text { de datos }\end{array}$ & $\begin{array}{l}\text { Código de } \\
\text { ética en } \\
\text { investigació } \\
n\end{array}$ & $\begin{array}{l}\text { se presenta } \\
\text { comportamiento de los } \\
\text { flujos de medicamentos } \\
\text { hacia las IPS para los dos } \\
\text { escenarios. Estos valores } \\
\text { corresponden a la cantidad } \\
\text { promedio mensual de } \\
\text { medicamentos, resultado } \\
\text { de la simulación de los dos } \\
\text { escenarios. En términos } \\
\text { porcentuales para el } \\
\text { escenario 1 se tiene que el } \\
66,83 \% \text { de los } \\
\text { medicamentos se } \\
\text { adquieren a se presenta el } \\
\text { comportamiento de los } \\
\text { flujos de medicamentos } \\
\text { hacia las IPS para los dos }\end{array}$ & $\begin{array}{l}\text { La simulación de escenarios de coordinación para la red de } \\
\text { suministro de medicamentos oncológicos, permite visualizar } \\
\text { alternativas beneficiosas para la gestión de abastecimiento en } \\
\text { las instituciones prestadoras de servicios de salud IPS. Los } \\
\text { resultados en los niveles de cumplimiento de la programación } \\
\text { de dosis del } 98,10 \% \text { y } 97,80 \% \text { para cada uno de los dos } \\
\text { escenarios, evidencian que la aplicación de mecanismos de } \\
\text { coordinación de información compartida y la toma conjunta } \\
\text { de decisiones mejora el comportamiento de los flujos de } \\
\text { suministro de medicamentos en las diferentes instancias de la } \\
\text { red, reduciendo el riesgo de desabastecimiento y por lo tanto } \\
\text { propiciando las condiciones de continuidad, cobertura y } \\
\text { eficiencia en la prestación de los servicios de oncología. La } \\
\text { evaluación de los dos escenarios que orientan el uso de } \\
\text { políticas y reglas de negociación y coordinación en la red de } \\
\text { suministro de medicamentos oncológicos, refleja el impacto } \\
\text { que a nivel de costo se puede presentar en el sistema de salud } \\
\text { colombiano. La reducción del } 13,99 \% \text { en el costo total en IPS }\end{array}$ \\
\hline
\end{tabular}


escenarios. Estos valores corresponden a la cantidad promedio mensual de medicamentos, resultado de la simulación de los dos escenarios. En términos porcentuales para el escenario 1 se tiene que el

$66,83 \%$ de los medicamentos se

adquieren a en el escenario 2, podría representar ahorros importantes en el sistema. La búsqueda de estrategias que prioricen la implementación de mecanismos de coordinación y negociación en la red de abastecimiento de medicamentos oncológicos, se constituye en una herramienta fundamental para la definición de políticas públicas y la toma de decisiones en el ámbito de la salud colombiana.

\begin{tabular}{|c|c|c|c|c|}
\hline 2. Autor & Año & Nombre de la Investigación & Revista donde se ubica la Publicación & Volumen Y Numero \\
\hline $\begin{array}{l}\text { Herrera, A. N., Guzmán, L. C., } \\
\text { \& Rodríguez, J. I. }\end{array}$ & 2020 & $\begin{array}{l}\text { Gestión de información de la } \\
\text { cadena de suministro de productos } \\
\text { perecederos: Aplicación de } \\
\text { BlockChain. }\end{array}$ & $\begin{array}{l}\text { Revista Ibérica De Sistemas e Tecnologías } \\
\text { Informaçã De Bogotá Colombia } \\
\text { https://search.proquest.com/docview/238 } \\
\text { 8304989?accountid=37408 }\end{array}$ & $\begin{array}{l}\text { 1051-1064. Retrieved } \\
\text { from }\end{array}$ \\
\hline
\end{tabular}

\section{CONTENIDO DE LA PUBLICACIÓN}

\begin{tabular}{|c|c|c|c|c|c|}
\hline $\begin{array}{l}\text { Tipo y Diseño de } \\
\text { Investigación }\end{array}$ & $\begin{array}{c}\text { Población y } \\
\text { Muestra }\end{array}$ & Instrumentos & Aspectos ético & Resultados & Conclusión \\
\hline $\begin{array}{l}\text { Descriptiva } \\
\text { cuantitativa }\end{array}$ & $\begin{array}{l}\text { Artículos } \\
\text { científicos }\end{array}$ & $\begin{array}{c}\text { Ficha de registro de } \\
\text { artículos }\end{array}$ & $\begin{array}{c}\text { Código de ética } \\
\text { en } \\
\text { investigación }\end{array}$ & $\begin{array}{l}\text { Las cadenas de suministro } \\
\text { de productos perecederos } \\
\text { son especialmente } \\
\text { vulnerables a incurrir en } \\
\text { pérdidas por una } \\
\text { inadecuada gestión de } \\
\text { información. Uno de los } \\
\text { factores más relevantes } \\
\text { dentro de este tipo de } \\
\text { cadenas es el tiempo, } \\
\text { debido a la vida útil } \\
\text { limitada del producto, si no } \\
\text { hay una coordinación }\end{array}$ & $\begin{array}{l}\text { Las soluciones de trazabilidad que ofrece } \\
\text { BlockChain fomentan el desarrollo de } \\
\text { prácticas comerciales basadas en } \\
\text { responsabilidad social y ambiental, } \\
\text { proporciona transparencia en el recorrido del } \\
\text { producto generando valor agregado gracias a la } \\
\text { confiabilidad, no solo para el cliente que lo } \\
\text { demanda sino para todos los agentes que } \\
\text { participan dentro de la cadena. } \\
\text { implementación de estas tecnologías permite } \\
\text { generar acciones preventivas, por medio de } \\
\text { históricos estableciendo pronósticos que dan } \\
\text { herramientas para crear estrategias de }\end{array}$ \\
\hline
\end{tabular}


eficiente entre los actores se pueden presentar retrasos que ocasionan desperdicios. Herramientas como el IoT y el BlockChain facilita que los agentes se comuniquen en tiempo real y que los datos administrados estén protegidos sin posibilidad de fraude, permitiendo la fluidez, la eficiencia y la confiabilidad de información que transita lo largo de la cadena. contingencia y acciones correctivas, disminuyendo el impacto de situaciones emergentes. Para la aplicación de BlockChain se están desarrollando productos de software a nivel mundial y se evidencia la rapidez con la que avanza gracias a la inversión que se realiza en algunas organizaciones, sin embargo, para su aplicación masiva, hace falta que estos desarrollos estén al alcance de más empresas que aún no lo consideran una opción por el desconocimiento que se tiene de la tecnología y debido a la desconfianza que representa la compartir información relevante para una organización. 


\begin{tabular}{|c|c|c|c|c|}
\hline 3. Autor & Año & Nombre de la Investigación & Revista donde se ubica la Publicación & Volumen Y Numero \\
\hline $\begin{array}{c}\text { Balcázar D, López C, \& } \\
\text { Wilson, A. }\end{array}$ & 2016 & $\begin{array}{l}\text { Lineamientos estratégicos para } \\
\text { coordinación en la cadena de } \\
\text { suministro de medicamentos y } \\
\text { propuesta de un modelo } \\
\text { matemático para medir los efectos } \\
\text { de la coordinación. }\end{array}$ & $\begin{array}{l}\text { Dyna. Bogotá Colombia. } \\
\text { https://search.proquest.com/docview/1 } \\
\text { 802641630?accountid=37408 }\end{array}$ & $\begin{array}{l}\text { 83(197), 203. Retrieved } \\
\text { from }\end{array}$ \\
\hline
\end{tabular}

CONTENIDO DE LA PUBLICACIÓN

\begin{tabular}{|c|c|c|c|c|c|}
\hline $\begin{array}{l}\text { Tipo y Diseño de } \\
\text { Investigación }\end{array}$ & $\begin{array}{c}\text { Población y } \\
\text { Muestra }\end{array}$ & Instrumentos & $\begin{array}{l}\text { Aspectos } \\
\text { ético }\end{array}$ & Resultados & Conclusión \\
\hline Descriptiva & $\begin{array}{c}\text { Base de } \\
\text { datos }\end{array}$ & $\begin{array}{l}\text { Ficha de } \\
\text { recolección de } \\
\text { datos }\end{array}$ & $\begin{array}{l}\text { Código de } \\
\text { ética en } \\
\text { investigación }\end{array}$ & $\begin{array}{l}\text { Para ejecutar el modelo, se } \\
\text { cumplen los siguientes supuestos: } \\
\text { 1). Los costos fijos y variables del } \\
\text { proveedor } 1 \text { son más altos que los } \\
\text { del proveedor } 2 \text { y, a su vez, los } \\
\text { costos del proveedor } 3 \text { son más } \\
\text { altos que los del proveedor } 2.2 \text { ) } \\
\text { Los costos de mantener los } \\
\text { medicamentos typep en inventario } \\
\text { son } 4,2 \text { y } 1 \text { por ciento del precio } \\
\text { del medicamento correspondiente } \\
\text { para los proveedores } 1,2 \text { y } 3 \text {, } \\
\text { respectivamente. 3) El costo de } \\
\text { mantener un medicamento tipo p } \\
\text { en el inventario de IPS es } 80 \% \text { del } \\
\text { precio del producto, ya que un } \\
\text { medicamento adecuado para uno el } \\
\text { paciente rara vez es apropiado para } \\
\text { otro. 4) El espacio para almacenar }\end{array}$ & $\begin{array}{l}\text { Para demostrar que la planificación coordinada } \\
\text { puede mejorar el rendimiento de la cadena de } \\
\text { suministro, el modelo matemático de programación } \\
\text { lineal entera mixta, presentada en la sección 5, e } \\
\text { usó para comparar dos escenarios con demanda } \\
\text { variable distribuciones en el mismo horizonte de } \\
\text { planificación. La atención al paciente es realizada } \\
\text { bajo el supuesto de coordinación que involucra un } \\
\text { libre flujo de información y cooperación para } \\
\text { satisfacer la demanda en el primer escenario; y el } \\
\text { supuesto de no cooperación en el segundo } \\
\text { escenario, donde la demanda excede la capacidad y } \\
\text { debe ser diferido. Se utilizaron datos emulados que } \\
\text { se asemejan a datos reales para no comprometer los } \\
\text { datos privados de la organización. }\end{array}$ \\
\hline
\end{tabular}




\begin{tabular}{|c|c|c|c|c|c|c|}
\hline 4. Autor & Año & \multicolumn{2}{|c|}{ Nombre de la Investigación } & \multicolumn{2}{|c|}{ Revista donde se ubica la Publicación } & Volumen Y Numero \\
\hline $\begin{array}{c}\text { Zúñiga, F., Luza, D., Soto, P., } \\
\text { \& Piñones, G. }\end{array}$ & 2015 & \multicolumn{2}{|c|}{$\begin{array}{l}\text { Propuesta de abastecimiento } \mathrm{d} \\
\text { medicamentos coordinand } \\
\text { multiniveles de demanda. un cas } \\
\text { ilustrativo chileno. }\end{array}$} & \multicolumn{2}{|c|}{$\begin{array}{c}\text { Estudios Gerenciales. Cali Colombia. } \\
\text { doi:http://dx.doi.org/10.1016/j.estger.2015 } \\
.08 .001\end{array}$} & Vol:31(137), 419-431. \\
\hline \multicolumn{7}{|c|}{ CONTENIDO DE LA PUBLICACIÓN } \\
\hline $\begin{array}{c}\text { Tipo y Diseño de } \\
\text { Investigación }\end{array}$ & $\begin{array}{c}\text { Población y } \\
\text { Muestra }\end{array}$ & $\begin{array}{c}\text { Instrumento } \\
\mathrm{s} \\
\end{array}$ & $\begin{array}{l}\text { Aspectos } \\
\text { ético }\end{array}$ & Resultados & \multicolumn{2}{|c|}{ Conclusión } \\
\hline $\begin{array}{l}\text { Descriptiva, } \\
\text { estudio de casos }\end{array}$ & & $\begin{array}{l}\text { Base de } \\
\text { datos }\end{array}$ & $\begin{array}{l}\text { Código de } \\
\text { ética en } \\
\text { investigación }\end{array}$ & $\begin{array}{l}\text { Los resultados } \\
\text { generados muestran } \\
\text { que la política } \\
\text { propuesta } \\
\text { disminuiría } \\
\text { semanalmente en un } \\
8,22 \% \text { los costos de } \\
\text { compra, en un } \\
95,47 \% \text { los costos de } \\
\text { ordenar y en un } \\
54,6 \% \text { los costos } \\
\text { totales }\end{array}$ & $\begin{array}{l}\text { El presente trabajo ha } \\
\text { establecer una política de } \\
\text { los multiniveles que ope } \\
\text { chilena en torno a la pr } \\
\text { insumos médicos en este } \\
\text { salud en Chile. El ahorro } \\
\text { compra de productos en } \\
\text { de un } 95,47 \% \text { en los cost } \\
\text { nivel generan un cuanti } \\
\text { coordinado completo. } \\
\text { magnitud de contar cor } \\
\text { abastecimiento y reit } \\
\text { seleccionar el control de } \\
\text { no tratando a todos los } \\
\text { centrándose en inventario } \\
\text { presente trabajo solo se } \\
\text { producían el } 80 \% \text { de los } \\
\text { extrapolar los resultados } \\
\text { productos que pertenece } \\
\text { con el fin de tener un por } \\
\text { proporcionalmente aun } \\
\text { derivado de la optimizac } \\
\text { aplicación de la política d } \\
\text { propuesta. }\end{array}$ & $\begin{array}{l}\text { nostrado la necesidad de } \\
\text { bastecimiento que coordine } \\
\text { an actualmente en la APS } \\
\text { visión de medicamentos e } \\
\text { ipo de establecimientos de } \\
\text { l } 8,22 \% \text { en los costos de la } \\
\text { dega central y la reducción } \\
\text { de ordenar en este mismo } \\
\text { so ahorro para el sistema } \\
\text { tos resultados resaltan la } \\
\text { este tipo de políticas de } \\
\text { an la importancia de } \\
\text { sstos en productos críticos, } \\
\text { productos por igual sino } \\
\text { críticos. Debido a que en el } \\
\text { cuparon los productos que } \\
\text { stos de compra, es posible } \\
\text { para cubrir el total de los } \\
\text { al surtido de inventarios, } \\
\text { ntaje de ahorro económico } \\
\text { nayor al propuesto, esto } \\
\text { in de costos lograda por la } \\
\text { abastecimiento coordinada }\end{array}$ \\
\hline
\end{tabular}




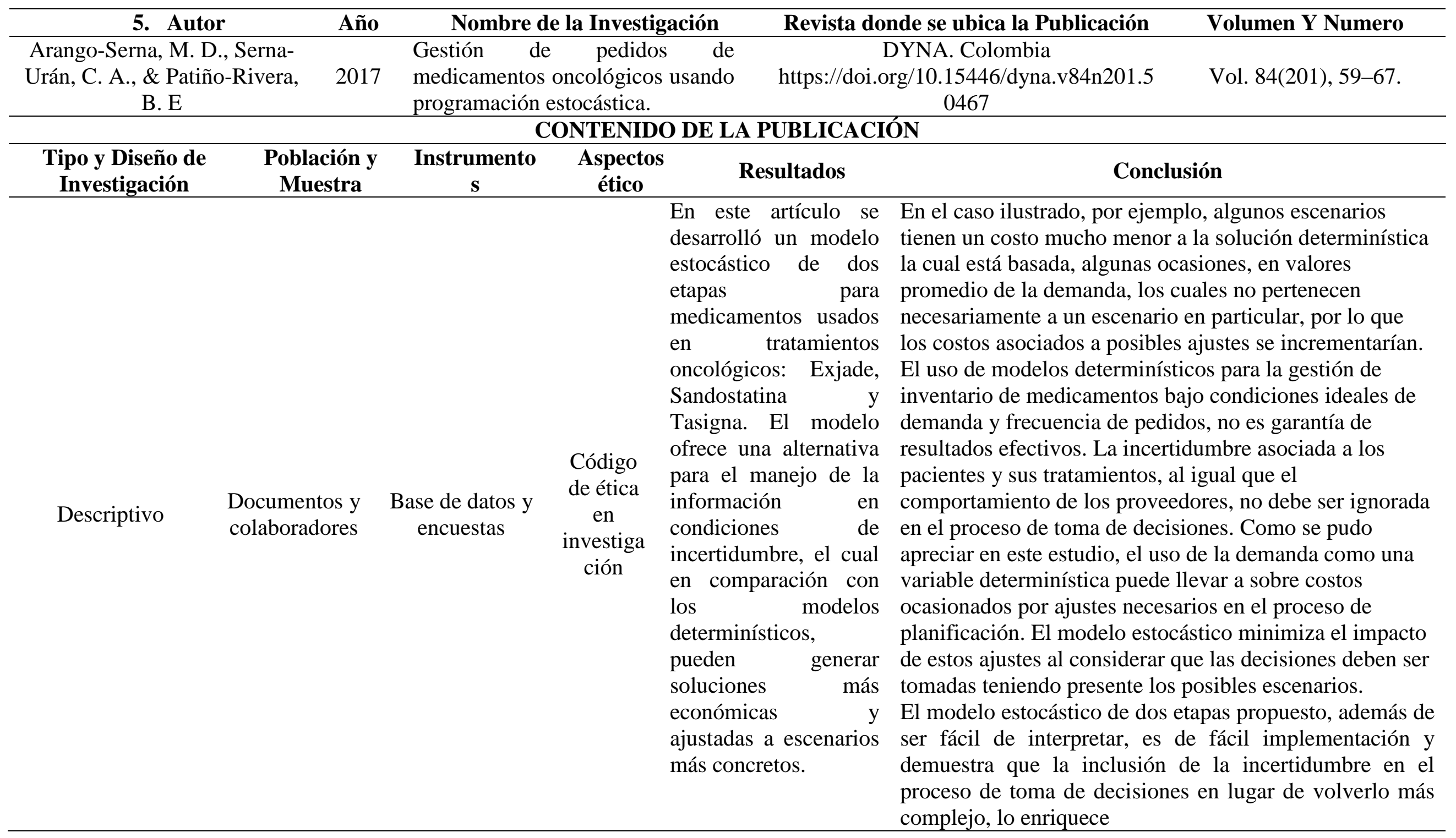




\begin{tabular}{|c|c|c|c|c|}
\hline 6. Autor & Año & \multicolumn{2}{|c|}{ Nombre de la Investigación } & Revista donde se ubica la Publicación \\
\hline \multicolumn{2}{|c|}{$\begin{array}{l}\text { Hernández O, Torres K, Ávila } \\
\text { E, Livingston J, \& Pinzón M. }\end{array}$} & \multicolumn{2}{|c|}{$\begin{array}{l}\text { Consumos y Costos de } \\
\text { Medicamentos: Herramienta para } \\
\text { la Gestión de Suministro de } \\
\text { Servicio Farmacéutico. }\end{array}$} & $\begin{array}{l}\text { Ciencia e Innovación En Salud. Colombia } \\
\text { https://doi.org/10.17081/innosa.3.1.237 }\end{array}$ \\
\hline \multicolumn{5}{|c|}{ CONTENIDO DE LA PUBLICACIÓN } \\
\hline $\begin{array}{l}\text { Tipo y Diseño de } \\
\text { Investigación }\end{array}$ & $\begin{array}{l}\text { Población } \\
\text { y Muestra }\end{array}$ & $\begin{array}{l}\text { Instrumento } \\
\mathbf{s}\end{array}$ & $\begin{array}{l}\text { Aspectos } \\
\text { ético }\end{array}$ & Conclusión \\
\hline $\begin{array}{l}\text { Descriptiva exploratoria } \\
\text { retrospectivo }\end{array}$ & $\begin{array}{l}\text { Document } \\
\text { os de } \\
\text { costo }\end{array}$ & $\begin{array}{l}\text { Base de } \\
\text { datos }\end{array}$ & $\begin{array}{l}\text { Código de } \\
\text { ética en } \\
\text { investigación }\end{array}$ & 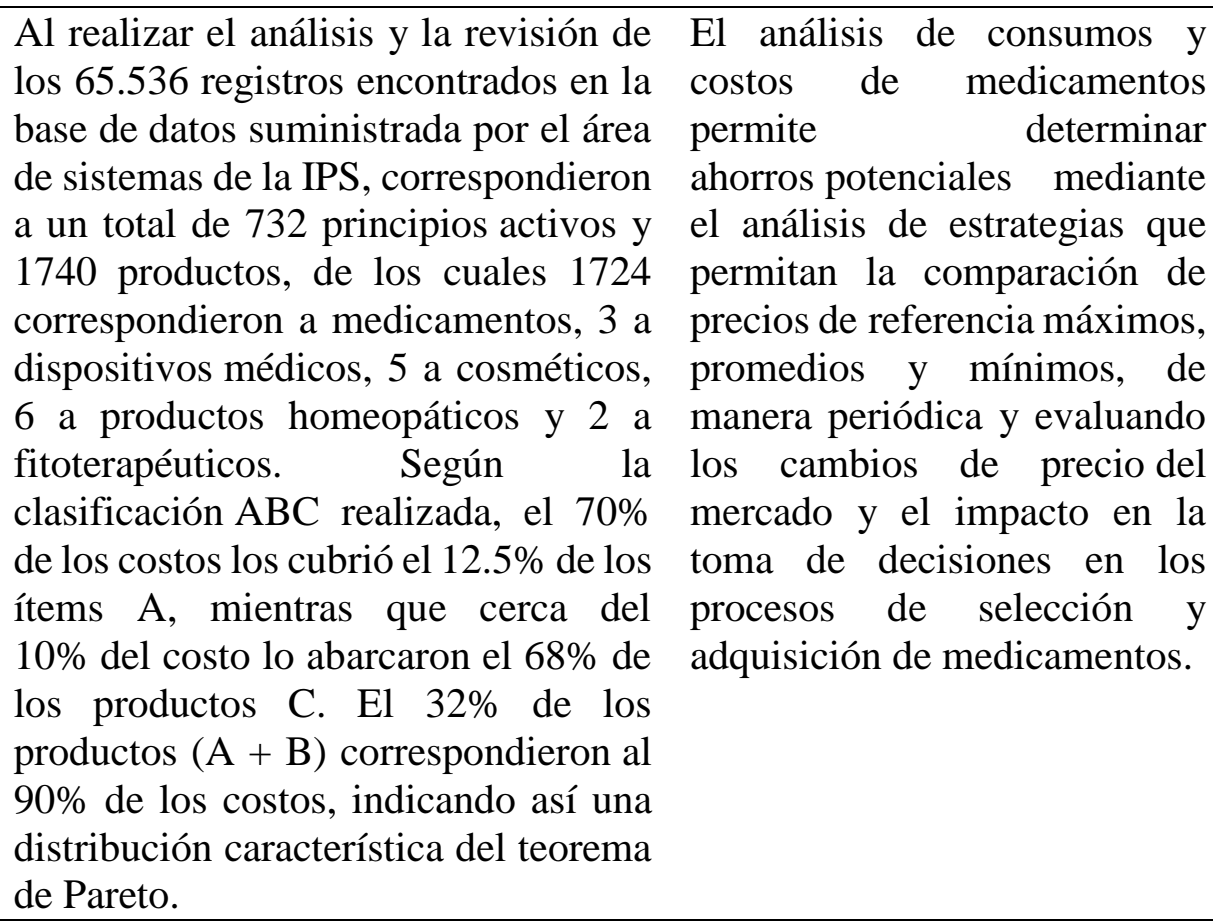 \\
\hline
\end{tabular}




\begin{tabular}{|c|c|c|c|c|}
\hline 7. Autor & Año & Nombre de la Investigación & \multirow{2}{*}{$\begin{array}{c}\text { Revista donde se ubica la Publicación } \\
\text { Cuadernos Latinoamericanos de } \\
\text { Administración. Cuba. } \\
\text { https://doi.org/10.18270/cuaderlam.v15i2 } \\
8.2685 \\
\end{array}$} & Volumen Y Numero \\
\hline $\begin{array}{c}\text { López T, Acevedo D., \& Peña } \\
\text { C. }\end{array}$ & 2019 & $\begin{array}{l}\text { La cadena de sumini } \\
\text { medicamentos en Cuba. }\end{array}$ & & $\begin{array}{l}\text { inoamericanos de } \\
\text { ación. Cuba. } \\
8270 / \text { cuaderlam.v15i2 } \\
2685\end{array}$ \\
\hline \multicolumn{5}{|c|}{ CONTENIDO DE LA PUBLICACIÓN } \\
\hline $\begin{array}{l}\text { Tipo y Diseño de } \\
\text { Investigación }\end{array}$ & $\begin{array}{l}\text { Población } \\
\text { y Muestra }\end{array}$ & $\begin{array}{cc}\text { Instrumento } & \begin{array}{c}\text { Aspect } \\
\text { s }\end{array} \\
\text { os ético }\end{array}$ & Resultados & Conclusión \\
\hline $\begin{array}{l}\text { Descriptiva exploratoria, } \\
\text { estudio de caso }\end{array}$ & $\begin{array}{l}\text { Bases de } \\
\text { datos }\end{array}$ & $\begin{array}{l}\text { Encuestas- } \\
\text { Cuestionario } \\
\text { s y guías de } \\
\text { entrevista }\end{array}$ & $\begin{array}{l}\text { Se seleccionó a la variable } \\
\text { disponibilidad, como el } \\
\text { indicador de impacto de la } \\
\text { cadena de medicamentos, } \\
\text { reafirmándose EMCOMED, } \\
\text { como la entidad } \\
\text { coordinadora de esta red, que } \\
\text { se encuentra en la Etapa II, } \\
\text { centrada en la } \\
\text { organización de la } \\
\text { integración de la } \\
\text { cadena de suministro. } \\
\text { Asimismo, a partir del } \\
\text { análisis de las } \\
\text { variables de coordinación y su } \\
\text { diagnóstico, fueron } \\
\text { propuestos la } \\
\text { proyectos de desarrollo } \\
\text { enfocados a consolidar } \\
\text { la gestión integrada y la } \\
\text { incorporación de tecnologías } \\
\text { de información en la misma. }\end{array}$ & $\begin{array}{l}\text { Se aplicó la retroalimentación del Procedimiento de } \\
\text { Desarrollo de Cadenas de Suministro para actualizar } \\
\text { el desarrollo de la cadena. Se identificó la cadena de } \\
\text { suministro: Medicamentos y productos asociados a } \\
\text { servicios médicos, siendo EMCOMED la entidad } \\
\text { coordinadora de la cadena. Se selecciona como } \\
\text { indicador de impacto la disponibilidad de productos, } \\
\text { permitiendo evaluar el objetivo } \\
\text { definido en la cadena, a partir de medir el } \\
\text { cumplimiento de pedidos realizados por } \\
\text { medicamento por las } \\
\text { instituciones de salud a los centros de distribución, } \\
\text { dando como resultado un } 31 \% \text { de pedidos completos } \\
\text { en } \\
\text { el período analizado. } \\
\text { La problemática fundamental radica, en la deficiente } \\
\text { integración entre los eslabones de la cadena, lo cual } \\
\text { se refleja en la baja disponibilidad en los puntos de } \\
\text { consumo. } \\
\text { La aplicación del MRL obtuvo una valoración } \\
\text { general de } 3.65 / 5.00 \text { puntos, con una evaluación } \\
\text { regular en }\end{array}$ \\
\hline
\end{tabular}




\begin{tabular}{|c|c|c|c|c|}
\hline 8. Autor & Año & Nombre de la Investigación & Revista donde se ubica la Publicación & Volumen Y Numero \\
\hline $\begin{array}{c}\text { Tobón-Marulanda, F., Loaiz } \\
\text { Ocampo, J., \& Rojas- } \\
\text { Durango, Y. }\end{array}$ & 2016 & $\begin{array}{l}\text { Evaluación de la gestión de } \\
\text { suministro de medicamento } \\
\text { antirretrovirales en una } \\
\text { institución prestadora de saluc } \\
\text { Medellín-Colombia } 2013 \text {. }\end{array}$ & $\begin{array}{c}\text { Revista Médicas UIS. Bucaramanga } \\
\text { Colombia } \\
\text { https://doi.org/10.18273/revmed.v29n2- } \\
2016001\end{array}$ & Vol. 29 Issue 2, p11-20. 10p. \\
\hline \multicolumn{5}{|c|}{ CONTENIDO DE LA PUBLICACIÓN } \\
\hline $\begin{array}{l}\text { Tipo y Diseño de } \\
\text { Investigación }\end{array}$ & $\begin{array}{l}\text { Población } \\
\text { y Muestra }\end{array}$ & $\begin{array}{c}\text { Instrumento } \\
\mathbf{S}\end{array}$ & Resultados & Conclusión \\
\hline $\begin{array}{l}\text { cualitativo retrospectivo } \\
\text { con enfoque hermenéutico }\end{array}$ & $\begin{array}{l}\text { Revisión y } \\
\text { análisis de } \\
\text { protocolos } \\
\text { del } \\
\text { sistema de } \\
\text { gestión de } \\
\text { la calidad } \\
\text { de la } \\
\text { institución }\end{array}$ & $\begin{array}{l}\text { Código de } \\
\text { ética en } \\
\text { investigación }\end{array}$ & $\begin{array}{l}\text { se encontró que la gestión del suministro de los } \\
\text { antirretrovirales en general se cumple conforme } \\
\text { a las normas. No obstante, se halló que el } \\
\text { seguimiento fármaco-terapéutico a cada uno de } \\
\text { los pacientes que asisten a la institución es } \\
\text { deficiente. Se evidenció que en el primer } \\
\text { trimestre del año } 201397,5 \% \text { de los pacientes } \\
\text { tratados con antirretrovirales, no le hicieron } \\
\text { seguimiento fármaco-terapéutico en } \\
\text { conformidad a la normatividad vigente y a lo } \\
\text { establecido por los protocolos }\end{array}$ & $\begin{array}{l}\text { se requiere mejorar y fortalecer } \\
\text { el servicio de atención } \\
\text { farmacéutico, liderado por } \\
\text { profesionales suficientes y } \\
\text { capacitados, que implementen } \\
\text { otro seguimiento fármaco- } \\
\text { terapéutico holístico, } \\
\text { sistemático y permanente }\end{array}$ \\
\hline
\end{tabular}




\begin{tabular}{|c|c|c|c|}
\hline 9. Autor & Año & Nombre de la Investigación & Revista donde se ubica la Publicación \\
\hline Ibañez, K. & 2014 & $\begin{array}{l}\text { Intervención farmacéutica sobre } \\
\text { la gestión de suministro de } \\
\text { medicamentos en la microred de } \\
\text { salud "El Bosque" de Trujillo, } \\
\text { Perú. }\end{array}$ & $\begin{array}{l}\text { Revista ciencia y tecnología. Trujillo } \\
\text { Perú. } \\
\text { http://search.ebscohost.com/login.aspx?di } \\
\text { rect=true\&db=edsbas\&AN=edsbas.A23D } \\
\text { 1EFD\&lang=es\&site=eds-live }\end{array}$ \\
\hline \multicolumn{4}{|c|}{ CONTENIDO DE LA PUBLICACIÓN } \\
\hline $\begin{array}{l}\text { Tipo y Diseño de } \\
\text { Investigación }\end{array}$ & $\begin{array}{l}\text { Población } \\
\text { y Muestra }\end{array}$ & $\begin{array}{c}\text { Instrumento } \\
\mathbf{S}\end{array}$ & Conclusión \\
\hline $\begin{array}{c}\text { Cuantitativo Pre } \\
\text { experimental }\end{array}$ & $\begin{array}{l}\text { Personal } \\
\text { de } 11 \\
\text { farmacias } \\
\text { y usuarios }\end{array}$ & $\begin{array}{cc}\text { Pre test y } & \text { Código de } \\
\text { post test - } & \text { ética en } \\
\text { Programa de } & \text { investigación } \\
\text { capacitación }\end{array}$ & 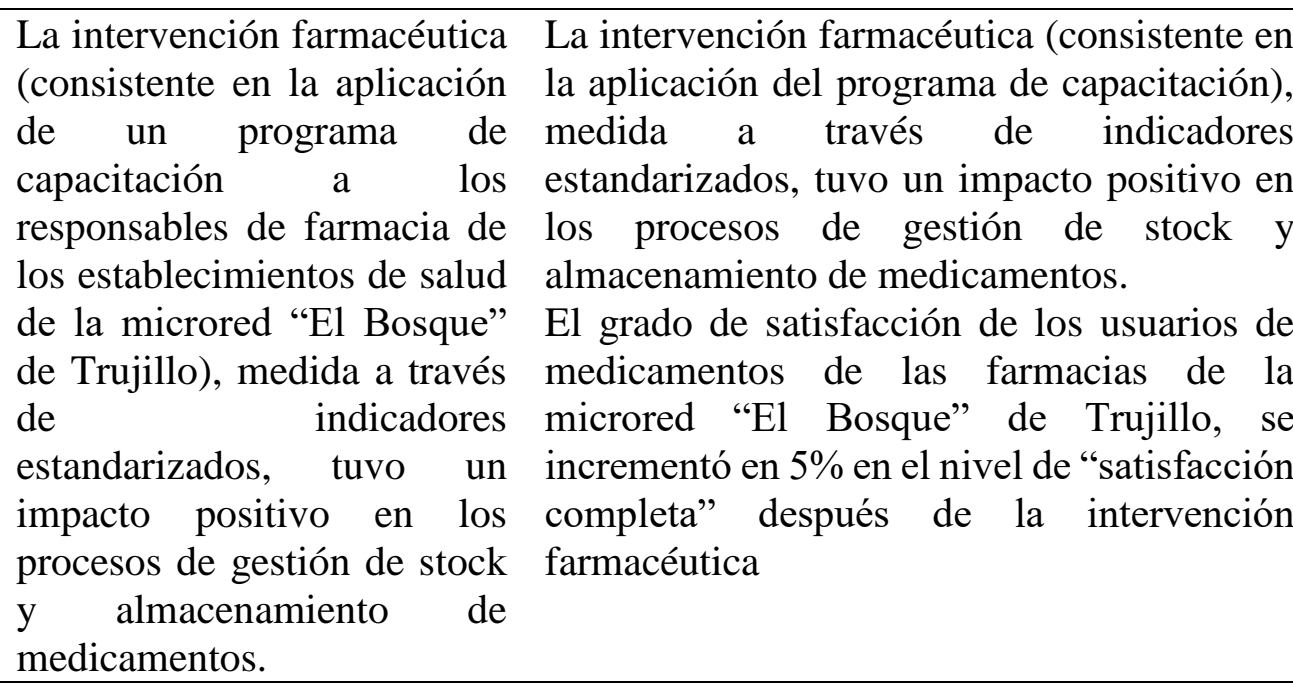 \\
\hline
\end{tabular}




\begin{tabular}{|c|c|c|c|c|}
\hline 10. Autor & Año & Nombre de la Investigación & Revista donde se ubica la Publicación & en Y Numero \\
\hline $\begin{array}{l}\text { Kuo, S., Ou, H.- } \\
\text { T., Wang, C.J.. }\end{array}$ & 2020 & $\begin{array}{l}\text { Gestión } r \text { Medicación } \\
\text { Suministro cadenas: } \\
\text { Lecciones aprendidas de } \\
\text { Taiwán durante la } \\
\text { planificación de la } \\
\text { pandemia y la preparación } \\
\text { COVID-19 para el futuro }\end{array}$ & $\begin{array}{c}\text { Anales de las Enfermedades Reumáticas } \\
\text { annrheumdis-2020-218223. EE.UU } \\
\text { https://www.scopus.com/record/display.uri?eid=2-s2.0- } \\
\text { 85090483568\&origin=resultslist\&sort=plff\&src=s\&st1=Medicatio } \\
\text { n+supply\&st2=\&sid=771c67b7e464b2f7cbe273beb892cc11\&sot= } \\
\text { b\&sdt=b\&sl=24\&s=TITLE\%28Medication+supply\%29\&relpos=5 } \\
\text { \&citeCnt=1\&searchTerm= }\end{array}$ & $2020-218223$ \\
\hline
\end{tabular}

\section{CONTENIDO DE LA PUBLICACIÓN}

\begin{tabular}{|c|c|c|c|c|c|}
\hline $\begin{array}{c}\text { Tipo y Diseño de } \\
\text { Investigación }\end{array}$ & $\begin{array}{l}\text { Población } \\
\text { y Muestra }\end{array}$ & $\begin{array}{c}\text { Instrume } \\
\text { ntos }\end{array}$ & $\begin{array}{l}\text { Aspectos } \\
\text { ético }\end{array}$ & Resultados & Conclusión \\
\hline $\begin{array}{c}\text { Cuantitativo } \\
\text { descriptivo }\end{array}$ & $\begin{array}{l}\text { Personal } \\
\text { de } \\
\text { farmacias } \\
\text { y usuarios }\end{array}$ & $\begin{array}{l}\text { Encuesta } \\
\text { Cuestionar } \\
\text { io }\end{array}$ & $\begin{array}{l}\text { Código de } \\
\text { ética en } \\
\text { investigación }\end{array}$ & $\begin{array}{l}\text { La escasez de medicamentos } \\
\text { y describe las } \\
\text { recomendaciones basadas en } \\
\text { evidencia sobre cómo } \\
\text { garantizar un suministro } \\
\text { estable de medicamentos y } \\
\text { mejorar la calidad y la } \\
\text { seguridad de los } \\
\text { medicamentos. Muchas } \\
\text { carencias de medicamentos se } \\
\text { han centrado en las } \\
\text { deficiencias de la fabricación } \\
\text { en el extranjero, pero el efecto } \\
\text { de la crisis COVID-19 en la } \\
\text { mala asignación de } \\
\text { medicamentos dentro de las } \\
\text { cadenas de suministro interna } \\
\text { de la nación también es una } \\
\text { gran preocupación }\end{array}$ & $\begin{array}{l}\text { La escasez de medicamentos y describe las recomendaciones } \\
\text { basadas en evidencia sobre cómo garantizar un suministro } \\
\text { estable de medicamentos y mejorar la calidad y la seguridad } \\
\text { de los medicamentos. Muchas carencias de medicamentos se } \\
\text { han centrado en las deficiencias de la fabricación en el } \\
\text { extranjero, pero el efecto de la crisis COVID-19 en la mala } \\
\text { asignación de medicamentos dentro de las cadenas de } \\
\text { suministro interna de la nación también es una gran } \\
\text { preocupación. Una amplia gama de partes interesadas } \\
\text { participa en las cadenas de suministro farmacéuticas, } \\
\text { incluidos reguladores gubernamentales, aseguradoras de } \\
\text { atención médica, compañías farmacéuticas, médicos y } \\
\text { farmacéuticos de primera línea, pacientes y familias, } \\
\text { asociaciones o sindicatos profesionales y de pacientes, e } \\
\text { incluso personas que adquieren medicamentos del extranjero. } \\
\text { Los insumos y esfuerzos colaborativos de todas estas partes } \\
\text { interesadas interdependientes son fundamentales para } \\
\text { establecer planes transparentes de preparación y contingencia } \\
\text { para abordar la escasez de medicamentos afectada por las } \\
\text { interrupciones de }\end{array}$ \\
\hline
\end{tabular}

$$
\text { DOI:10.37811/ p. } 1197
$$




\section{DISCUSIÓN}

Posterior a la revisión sistemática de los artículos, el 100\% corresponden a América latina y El Caribe, resaltando el país de Colombia con 70\%, seguido de Perú, Cuba y Estados Unidos de América con 10\% respectivamente.

En relación a la variable, podemos menciar a Aguas, J., Jaimes, W., \& Escamilla, E., quienes concluyeron que, La simulación de escenarios de coordinación para la red de suministro de medicamentos oncológicos, permite visualizar alternativas beneficiosas para la gestión de abastecimiento en las instituciones prestadoras de servicios de salud IPS. También podemos mencionar a Herrera, A, Guzmán, L., \& Rodríguez, J., concluyen que, las cadenas de suministro de productos perecederos son especialmente vulnerables a incurrir en pérdidas por una inadecuada gestión de información. Asimismo, Balcázar D, López C, \& Wilson, A, quienes establecen que, para demostrar que la planificación coordinada puede mejorar el rendimiento de la cadena de suministro, el modelo matemático de programación lineal entera mixta, presentada en la sección. Igualmente, Zúñiga, F., Luza, D., Soto, P., \& Piñones, G., establecen que, se demostró la necesidad una política de abastecimiento que coordine los multiniveles que operan actualmente en la APS chilena en torno a la provisión de medicamentos e insumos médicos en este tipo de establecimientos de salud en Chile.

Por otro lado, Arango-Serna, M.., Serna-Urán, C. , \& Patiño-Rivera, B. , determinó que, algunos escenarios tienen un costo mucho menor a la solución determinística la cual está basada, algunas ocasiones, en valores promedio de la demanda, los cuales no pertenecen necesariamente a un escenario en particular, por lo que los costos asociados a posibles ajustes se incrementarían. Asimismo, Hernández O, Torres K, Ávila E, Livingston J, \& Pinzón M, concluyeron que, el análisis de consumos y costos de medicamentos permite determinar ahorros potenciales mediante el análisis de estrategias que permitan la comparación de precios de referencia máximos, promedios y mínimos, de manera periódica y evaluando los cambios de precio del mercado y el impacto en la toma de decisiones en los procesos de selección y adquisición de medicamentos. Además, López T, Acevedo D., \& Peña C., concluyen que, al aplicar la retroalimentación del Procedimiento de Desarrollo de Cadenas de Suministro para actualizar el desarrollo de la cadena. Se identificó la cadena de suministro: Medicamentos y productos asociados a 
servicios médicos, siendo EMCOMED la entidad coordinadora de la cadena. TobónMarulanda, F., Loaiza-Ocampo, J., \& Rojas-Durango, Y., establece que, se requiere mejorar y fortalecer el servicio de atención farmacéutico, liderado por profesionales suficientes y capacitados, que implementen otro seguimiento fármaco-terapéutico holístico, sistemático y permanente. También, Ibañez, K., La intervención farmacéutica (consistente en la aplicación del programa de capacitación), medida a través de indicadores estandarizados, tuvo un impacto positivo en los procesos de gestión de stock y almacenamiento de medicamentos. Sin embargo, Kuo, S., Ou, H.-T., Wang, C.J., establecen que, la escasez de medicamentos y describe las recomendaciones basadas en evidencia sobre cómo garantizar un suministro estable de medicamentos y mejorar la calidad y la seguridad de los medicamentos.

\section{CONCLUSIÓN O CONSIDERACIONES FINALES}

La revisión sistemática de los 10 artículos científicos, sobre estilos de consumo y vida sostenible en el contexto de la crisis económica, corresponde a diferentes bases de datos, todos ellos corresponden al tipo y diseño de estudios descriptivos.

Posterior a la revisión sistémica, se concluye que el 60\% de los estudios relacionados que los estilos de consumo influyen en la vida sostenible en el contexto de la crisis económica, debido a que los estilos de consumo demandan políticas, condiciones y consecuencias de contaminación básicamente; y se puede establecer que el consumo tiene un impacto social lógicamente distinto en los diferentes países del mundo. Por otro lado, el 50\% concluye que los estilos de vida son importantes porque el comportamiento de las personas depende de ello que establezca un adecuado consumo. Así mismo las consecuencias del consumo no disminuyó muy por lo contrario sino no tomamos atención va en incremento. Por otro lado, la promoción de la salud es una estrategia clave para dotar de competencias y capacidades a las personas para que el consumo tome un camino diferente a favor de la vida sostenible a pesar de la crisis económica de los países, porque hay acciones que no depende de dinero sino del comportamiento social de las personas.

Se recomienda un trabajo de equipo multidisciplinario y multisectorial para la mejorar el consumo y garantizar una vida sostenible para las nuevas generaciones 


\section{LISTA DE REFERENCIAS}

Resolución Ministerial No 116 - MINSA (2018). Que aprueba la Directiva Administrativa $\mathrm{N}^{\circ}$ 249/MINSA/2018 publicado el 15 febrero 2018, Capturado en http://www.digemid.minsa.gob.pe/UpLoad/UpLoaded/

PDF/Normatividad/2018/RM_116-2018.pdf.

Arias, F. (2012). El proyecto de investigación: Introducción a la metodología científica. (6 $6^{\circ}$ ed.). Venezuela, Caracas: Episteme.

Bernal, C. (2010). Metodología de la Investigación. $3^{\circ}$ ed. Colombia: Editorial Pearson.

Contreras, A. (2014). Evaluación y propuestas para mejorar la calidad del servicio entregado a los químicos farmacéuticos de farmacias cruz verde a través del servicio de información de medicamento, tesis para obtener el grado de magister en Gerencia de los Servicios de Salud en la Universidad Austral de Chile

Digemid (2016), Dirección General de Medicamentos Insumos y Drogas, abastecimiento de medicamentos, http://www.digemid.minsa.gob.pe/

Espinoza, C. (2017) Planeamiento estratégico para el primer nivel de atención pública del sector salud de la Región Junín 2017. Tesis para obtener el grado de magíster pontificia universidad católica del Perú escuela de posgrado. Capturado http://tesis.pucp.edu.pe/repositorio/handle/123456789/9585

García O. Luis (2015). Metodología de la Investigación Clínica. Centro de Salud "La Alamedilla”. Salamanca, España.

Gómez, S. (2012). Metodología de la investigación. México: Red tercer milenio.

Hernández, R., Fernández, C. y Baptista, P. (2010). Metodología de la investigación.

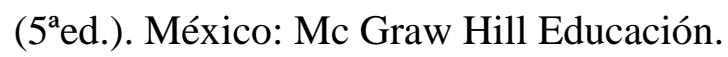

Hurtado, J. (2010). Metodología de la investigación: Guía para la comprensión holística de la ciencia. Caracas: Quirón-Sypal

Ministerio de Salud (2018). Normas Legales. RM No 116-2018/MINSA. Directiva 249/DIGEMID. Lima, Perú. Capturado http://www.digemid.minsa.gob.pe/Main.asp?Seccion=475

Ministerio de Salud (2017). Reglamento de Organización y Funciones. Lima, Perú. Capturado: http://www.minsa.gob.pe/transparencia/index.asp?op=107

Ministerio de Salud (2018) Indicadores de disponibilidad de medicamentos esenciales a 
junio

2018.

Lima,

Perú.

http://www.digemid.minsa.gob.pe/UpLoad/UpLoaded/PDF/Boletines/Indicadores/

B17_INDICADORES_2018-06_I_Nivel.pdf

Ministerio de Salud (2012) Propuesta modelo de gestión integral de suministro de medicamentos e insumos de salud, Ministerio de salud de El Salvador, organización panamericana de la salud / organización mundial de la salud, San Salvador 2012. Capturado:

https://www.paho.org/els/index.php?option=com_docman\&view=download\&categ ory_slug=medicamentos\&alias=1176-modelo-de-gestion-integral-de-suministrode-medicamentos-e-insumos-en-salud \&Itemid=364

Monje, C. (2011). Metodología de la investigación cuantitativa y cualitativa. Universidad Sur Colombia. Colombia. Neiva. Organización Mundial de la Salud. (2012). Definición de Salud. Capturado el 10 agostos 2018. http://teoriaoms.blogspot.com/2008/05/la-organizacin-mundial-de-la-salud-oms.html.

Organización Mundial de la Salud. (2012). Propuesta modelo de gestión integral de suministro de medicamentos e insumos de salud, Ministerio de Salud de El Salvador, organización panamericana de la salud / Capturado enhttps://www.paho.org/els/index.php?option=com_docman\&view=download\&cat egory_slug=medicamentos\&alias=1176-modelo-de-gestion-integral-de-suministrode-medicamentos-e-insumos-en-salud $\&$ Itemid=364

Pulgarin D. y Tabarquino R. (2015) Análisis del diseño de una red integradas servicios de salud

en

Colombia

file://C:/Users/Julio/Desktop/MAESTRIA\%20UCV/Tesis\%20relacionadas/Tesis $\% 20$ analisis $\% 20 \mathrm{del} \% 20 \mathrm{dise} \% \mathrm{C} 3 \% \mathrm{~B} 1 \mathrm{o} \% 20 \mathrm{de} \% 20$ una $\% 20$ red $\% 20 \mathrm{integra} \% 20$ salu d\%20Colombia.pdf

Quispe M. y Jorge L. (2017) “Gestión de abastecimiento de medicamentos y la calidad en los servicios de farmacia del hospital María Auxiliadora”. Tesis para obtener el grado de magister en Gestión de los Servicios de Salud en la Universidad César Vallejo, Lima-Perú.

Resolución Ministerial No 116 - MINSA (2018). Que aprueba la Directiva Administrativa $\mathrm{N}^{\circ}$ 249/MINSA/2018 publicado el 15 febrero 2018, Capturado en 
http://www.digemid.minsa.gob.pe/UpLoad/UpLoaded/

PDF/Normatividad/2018/RM_116-2018.pdf Saco, S. (1995) Declaración del alma ata.

Capturado

de:

http://sisbib.unmsm.edu.pe/bvrevistas/situa/1995_n5/declaracion.htm

Salazar, J. (2014). Factores de riesgo y la ruptura de stock de medicamentos en la Dirección Distrital de salud N 18D02-2014, tesis para obtener el grado de Maestría en Gestión local y políticas públicas en la Universidad Técnica de Ambato Ecuador. Seligman, M. (2015). Teoría del Bienestar o Modelo PERMA. Capturado el 22 de agosto 2018. http://www.crecimientopositivo.es/portal/teoria-del-bienestaro-modelo-perma-de-martin-seligman

Soto, O. (2014). Propuesta metodológica para la gestión del riesgo en las redes Abastecimiento. Caso de estudio abasto de medicamentos oncológicos de una IPS (Institución prestadora de salud) de Bogotá", tesis para optar el grado de Magíster en Ingeniería Industrial en la Universidad Nacional de Colombia.

Supo, J. (2014). Como empezar una tesis. Perú: Bioestadístico. EIRL

Tobar, F. (2002). Modelos de gestión en salud. Buenos Aires. Recuperado de: http://www.saludcolectiva-unr.com.ar/docs/SC-138.pdf

Urbina, A. (2015). "Modelo de gestión de calidad en el servicio de farmacia del Hospital Básico Privado Duran de la ciudad de Ambato en el año 2015 Ecuador”, tesis para obtener el grado de magister en Gerencia de los Servicios de Salud en la Universidad Regional Autónoma de los Andes Unidades

Zanipatin, B. (2011). "Elaboración de un Manual para el Suministro de Medicamentos" Riobamba de Ecuador, tesis para obtener el grado de magister en Gerencia de los Servicios de Salud en la Universidad Regional Autónoma de los Andes Unidad INRUD del curso OMS/INRUD titulado Promoting Rational Drug Use (Fomento del uso racional de medicamentos) 